University of Wollongong

Research Online

Faculty of Engineering and Information

Faculty of Engineering and Information

Sciences - Papers: Part A

Sciences

$1-1-2015$

\title{
File sharing in cloud computing using win stay lose shift strategy
}

Yilei Wang

Ludong University

Willy Susilo

University of Wollongong,wsusilo@uow.edu.au

Tao Li

Northeastern University, t1839@uow.edu.au

Qiuliang Xu

Shandong University

Follow this and additional works at: https://ro.uow.edu.au/eispapers

Part of the Engineering Commons, and the Science and Technology Studies Commons

Research Online is the open access institutional repository for the University of Wollongong. For further information contact the UOW Library: research-pubs@uow.edu.au 


\title{
File sharing in cloud computing using win stay lose shift strategy
}

\author{
Abstract \\ Traditionally, users in clouds are assumed to be willing to store and share files in clouds, where the \\ security and efficiency issues are discussed. However, few studies involve the incentives to share files in \\ clouds. In this paper, we delve into users' incentives for using the cloud system to store and share their \\ files towards the view of game theory. More specifically, the process of file sharing is reduced to an \\ infinitely repeated prisoner's dilemma PD game and the action of sharing files in clouds is considered as \\ the action of cooperation in the infinitely repeated PD game. We incorporate win stay lose shift WSLS \\ strategy into file sharing and simulate it compared with tit-for-tat TFT strategy in clouds. Simulation \\ results show that WSLS is an optimal strategy for users to share their files in clouds. Furthermore, WSLS \\ is robust for unintentional deviation and returns to mutual cooperation after deviation.

\section{Keywords} \\ cloud, sharing, file, win, shift, stay, strategy, computing, lose \\ Disciplines \\ Engineering | Science and Technology Studies

\section{Publication Details} \\ Wang, Y., Susilo, W., Li, T. \& Xu, Q. (2015). File sharing in cloud computing using win stay lose shift \\ strategy. International Journal of High Performance Computing and Networking, 8 (2), 154-166.
}


File Sharing in Cloud Computing Using Win Stay Lose

Shift Strategy

\title{
Yilei Wang ${ }^{1,2}$, Willy Susilo ${ }^{3}$, Tao $\mathbf{L i}^{1}$, Qiuliang $\mathbf{X u}^{2 *}$
}

1.School of Information and Electrical Engineering,

Ludong University, China

E-mail: wangyilei_2000@126.com

2.Department of Computer Science and Technology,

Shandong University, China

E-mail: xql@sdu.edu.cn

3.Centre for Computer and Information Security Research,

School of Computer Science and Software Engineering,

University of Wollongong,Australia

E-mail: wsusilo@uow.edu.au

*Corresponding author

\begin{abstract}
File sharing in clouds means that users store and share their files in clouds, which can greatly improve the utilization rate of the files. Traditionally, users in clouds are assumed to be willing to store and share their files in clouds, where the security and efficiency issues are discussed. However, little works involve the incentives for users to share files in clouds. That is, why user are willing to share files in clouds. In this paper, we delve into users' incentives for using the cloud system to store and share their files towards the view of game theory. More specifically, the process of file sharing is reduced to an infinitely repeated prisoner's dilemma (PD) game and the action of sharing files in clouds is considered as the action of cooperation in the infinitely repeated PD game. The main task of this paper is to find proper strategies such that users in clouds have incentives to share their files with others. To complete this task, we incorporate Win-Stay-Lose-Shift (WSLS) strategy into file sharing and simulate it compared with Tit-for-Tat (TFT) strategy in clouds. Simulation results show that WSLS is an optimal strategy for users to share their files in clouds. That is, the rounds needed for users to adopt cooperation when they take WSLS are fewer than those when they take TFT. Furthermore, WSLS is robust for unintentional deviation and returns to mutual cooperation after deviation. However, TFT is not robust for unintentional deviation. Thus WSLS is better than TFT for users to share files in clouds.
\end{abstract}

Keywords: File sharing, Win stay lose shift strategy, Game theory, Cloud computing.

\section{Biographical notes:}

Yilei Wang received her Master Degree from Shandong Normal University in 2004 and Doctoral Degree from Shandong University in 2014. Currently she is a teacher at Ludong University. Her research interests include secure protocol and rational secret sharing.

Willy Susilo received a Ph.D. in Computer Science from University of Wollongong, Australia. He is a Professor at the School of Computer Science and Software Engineering and the director of Centre for Computer and Information Security Research (CCISR) at the University of Wollongong. $\mathrm{He}$ is currently holding the prestigious ARC Future Fellow awarded by the Australian Research Council (ARC). His main research interests include cryptography and information security. His main contribution is in the area of digital signature schemes. He has served as a program committee member in dozens of international conferences. He has published numerous publications in the area of digital signature schemes and encryption schemes.

Tao Li received his Bachelor Degree from Dalian University of Technology in 2007. Currently he works at Ludong University as a lecture and his research interests include computer science and network security.

Qiuliang Xu is a Professor and doctoral supervisor of computer science and technology at Shandong University. Currently he is a sub-decanal of department of science and technology at Shandong University. He has long-term research on theoretical study of information security, cryptology etc. He has published more than 30 high-quality papers in the most famous academic publications in recent five years. Now he is a chair of Nation Science Foundation of China and other foundations. 


\section{Introduction}

Cloud computing refers to the usage, through Internet, of diverse applications as if they were installed in user's computer, independently of platform and location. Cloud computing environments are triggered by the widely used Internet connected systems and distributed applications. Users and clients purchase computing power whenever they need under cloud computing environments. Some web-based applications operate in the software as a service (SaaS) model, such as Google Docs and Customer Relationship Management Zhou et al. (2010). The frameworks and environments of cloud computing may undertake various issues in distributed and ubiquitous computing systems. The availability of infrastructure as a service (IaaS) and platform as a service (PaaS) environments are building blocks for cloud computing based applications. Furthermore, they also stimulate the research and development of technologies to support new applications. In a word, the emergence of cloud computing is a new revolution in IT technology since it reconstructs business models on the way IT industry working. Cloud storage is an evolving service-oriented paradigm, which provides new options for supporting data management functions with different economics and operational characteristics. One of the basic tasks for users is to easily access files stored in clouds. That is, cloud storage can offer a platform for users to share their data except storing. Data sharing is crucial to many collaborative works and can enhance data utilization ratio in clouds.

Previous works pay attention to the security issues in outsourcing data Ficco (2013); Xiao and Han (2014). However, the incentive for users to share is a basic problem in file or data sharing. If users have no incentives to share their data or store their data into cloud storage, the issue of discussing cloud storage security loses its significance. In this paper, we discuss the incentives for users to store or share their data in clouds by utilizing game theory. In game theory, users have the property of utilities and they will achieve different utilities according to whether they are willing to share their data. Towards the view of game theory, users wish to maximize their utilities when they interact with other users in clouds by taking optimal strategies. The main task of this paper is to find optimal strategies for users in clouds such that each of them has incentives to share data. In the following sections, we abuse the notion of file sharing as data sharing in clouds.

\subsection{Related works}

File sharings include architectures for enterprise usage $\mathrm{Li}$ et al. (2004); Schwartz (2007). However, these works do not consider the enterprise practice or collaboration problems. File sharing is widely used in various fields such as media sharing, computer network and protocol issues Christin et al. (2005); Johnson et al. (2009). Tang et al. propose the "LiveWire" and "Consolidarity" project using common files of enterprise users to make file sharing easier Tang et al. (2007). Whalen et al. put forward a user-centered process

Copyright (c) 2008 Inderscience Enterprises Ltd. which invents a prototype file manager revealing file sharing activity Whalen et al. (2008). There are also some filesharing systems. Voida et al. probe into the way users share, the faults in sharing, and how these faults reflect a gap in sharing practices supported by current sharing tools Voida et al. (2006). Rader analyzes difficult problems of shared files within a hierarchical filing structure Rader (2009) and problems of labeling files for an audience Rader (2010). Muller et al. discuss the role of "information curators" in file sharing systems and present better tools in larger file sharing services in order to manage groups of files Muller and Feinberg (2010). His sequence work presents several usage patterns for file sharing in the enterprise Muller et al. (2010). Shami et al. discuss the problem of social file sharing system for enterprise and provide a strong foundation for this problem Shami et al. (2011). Wang et al. propose a middleware "mCloud" between cloud storage and clients in order to provide data sharing service by using the technologies like virtualization, chunking and caching Wang et al. (2010). Canedo et al. stress on the problem of trust in file sharing within clouds environment Canedo et al. (2011). Xu et al. put forward a certificateless proxy re-encryption scheme for secure data sharing within public cloud Xu et al. (2012).

Game theory concerns with the mutual communications between participants, where they dedicated to understand the intricacies of collaborative interaction among players with conflicting interests. Game theory has been successfully applied to various problems such as congestion control, routing and networking Altman et al. (2006); Roughgarden (2010), fairly allocating resource for cloud computing services Ge et al. (2012); Niyato et al. (2011), detecting intrusion Lo et al. (2010), cheating in clouds computing Carroll and Grosu (2011); Hu et al. (2012) and cooperating to share files Yang et al. (2012) etc. Yang et al. studied cooperative communication Yang et al. (2012), where nodes in networks make decisions in line with their interests. Zhang et al. delve into the interactions among users within the file sharing applications. They incorporate the TFT strategy into the file sharing game such that a connection is set up when both users find this connection beneficial. Furthermore, they prove that Nash equilibrium exists in several games and find the loss of efficiency due to selfish behavior is inevitable even though TFT is considered as a panacea to prevent selfish behaviors. Game theory can also be used in secret sharing schemes, where rational parties only want to maximize their utilities Halpern and Teague (2004); Wang et al. (2014, 2013).

\subsection{Motivations and contributions}

The motivation of introducing rational file sharing derives from the intuition of rational secret sharing (RSS). In RSS, the crucial point is how to encourage parties to send others their shares, which just like cooperation with others in PD game. In fact, the utility of RSS scheme follows the definition in PD game. Since mutual cooperation rarely appears in one-shot PD game, there often need multiple rounds for rational parties to exchange their shares in RSS schemes. The multiple-round interactions in RSS are similar to infinitely iterated PD games. Therefore, we may use the strategies in infinitely iterated 
PD games about mutual cooperation to encourage parties to send others shares in RSS schemes. Previous works achieve various results about RSS Micali and Shelat (2009); Wang et al. (2012a,b).

RSS solves the problem how to securely share a secret among distrustful parties while file sharing in clouds solves the problem how to securely share files among distrustful parties. These two problems have some similarities. For example, they both discuss how to share something among distrustful parties considering security issues. Therefore, we can borrow the notion of rational parties from RSS to apply in file sharing on the basis of cloud. Rational parties in RSS are neither semi-honest nor malicious. They decide to participate in RSS only when cooperation can maximize their utility. Similarly, parties who want to share files in clouds are no longer simply assumed to be willing to share their files with other. That is, rational parties in clouds are not simply divided into two kinds, willing to share file or not willing to. Instead, they decide whether to share files according to their utilities. If sharing files can maximize their utility, then they share; Otherwise, not share. This property is similar to that of rational parties in RSS. Just like the scenario in RSS, the action cooperate (denoted as $C$ ) in file sharing means that parties are willing to share with others, while the action defect (denoted as $D$ ) means the opposite direction. The utility definition is also similar to RSS. To successfully share files in clouds and get optimal utility, the file sharing scheme should be designed such that parties are willing to adopt $C$. Therefore, there should be multiple rounds in files sharing. That is, the utility definition in RSS can directly apply in the scenario of file sharing in clouds. Furthermore, file sharing in clouds can also be considered as infinitely iterated PD games and its main task is still how to encourage mutual cooperation among parties in clouds. In other words, rational file sharing means that parties in clouds decide whether to share their files according to their utility and their main target is to maximize their utility. Fig. 1 presents the relationships between rational secret sharing and rational file sharing. They both use the utility definition derived from PD game and their main targets are both encourage cooperation among distrustful rational parties.

Recall that cooperation rarely appears in one shot PD game and multiple rounds are often introduced into rational file sharing in clouds. Therefore rational file sharing can be considered as infinitely iterated PD game. Towards the view of infinitely iterated PD game, there are many strategies to encourage mutual cooperation like TFT strategy Axelrod (1990). Axelrod conducted computer tournaments in order to find the optimal strategies. There were altogether 16 entries in the first round and 63 in the second round. The simplest TFT strategy won in both tournaments. The basic idea of TFT strategy is to make parties cooperate in the first round and then do whatever actions the opponents did in the previous round. The successful TFT strategy inspired subsequent researches. However, there are still two shortcomings.

1. TFT is not robust for noise. For example, two parties interact with each other, if one defects by mistake, then his opponent will defect in the following rounds.

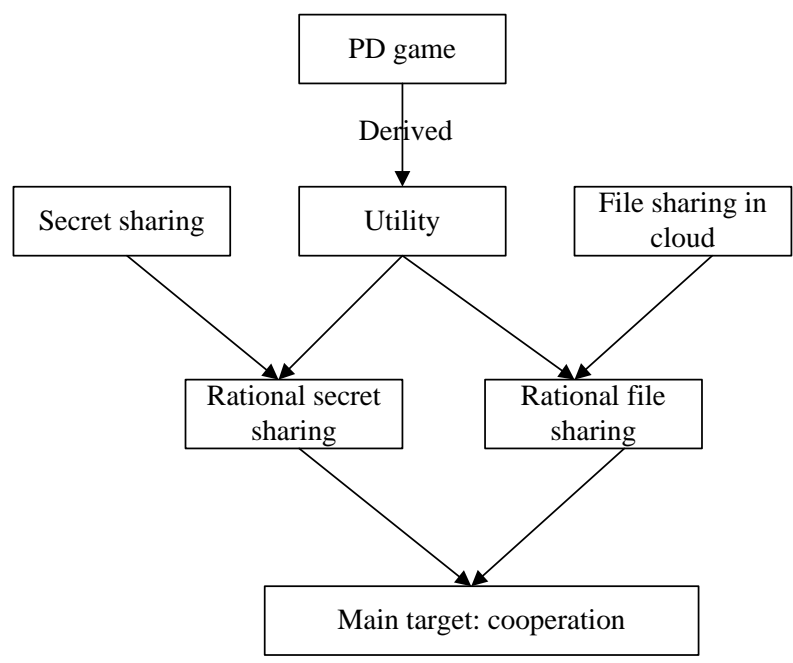

Figure 1 The relationships between rational secret sharing and rational file sharing.

Finally, mutual defect will be the result till the end of the protocol/game. TFT punishes any defect even it is unintentional. Once one mistake appears, they have no chance to go back to mutual cooperation. It is obviously undesirable for parties who wish to cooperate.

2. TFT is not evolutionarily stable. For example, suppose in clouds, some parties adopt cooperate strategy (CS) all through the rounds and some others adopt TFT strategy. CS and TFT have the same utility if there is no noise. However, the abundance of CS can increase by random shift. Once the abundance surpasses a certain threshold, parties will adopt defect strategy (DS) all through the rounds.

WSLS is put forward as a simple strategy to overcome the above two shortcomings Nowak and Sigmund (1993). The basic idea of WSLS for two parties' game is as the following.

1. If the outcome for the first party is $(C, C)$ or $(D, C)$ in the current round, then the first party will adopt the same action in the next round.

2. If the outcome for the first party is $(D, D)$ or $(C, D)$ in the current round, then the first party will adopt the opposite action in the next round.

Fig. 2 presents the cases where both parties use WSLS strategies. Fig. 3 presents the cases where both parties use TFT strategies. Fig. 4 presents the case where one party adopts TFT strategy and the other adopts WSLS strategy. In Fig. 2, 3 and 4, the sub-figures (a) denote that no parties deviate, (b) denote that the first party deviates unilaterally while the second party does not deviate, (c) denote that the second party deviates unilaterally while the first party does not deviate, and (d) denote both parties deviates simultaneously in the same round. From these figures, it is evident that WSLS can reach mutual cooperation when one or two parties adopt $D$ by mistake, while TFT can not correct this mistake till the end round. 


\begin{tabular}{|c|c|c|c|c|c|c|c|c|c|}
\hline \multirow[t]{2}{*}{ WSLS } & $\cdots \cdots \mathrm{C}$ & $\begin{array}{l}\mathrm{C} \\
\mathrm{C}\end{array}$ & $\begin{array}{l}\mathrm{C} \\
\mathrm{C} \\
\end{array}$ & $\begin{array}{l}\text { C } \\
\text { C }\end{array}$ & $\begin{array}{l}\text { C } \\
\mathrm{C} \\
\end{array}$ & C & $\begin{array}{l}\mathbf{C} \\
\mathrm{C} \\
\end{array}$ & $\begin{array}{l}\text { C } \\
\mathrm{C}\end{array}$ & $\begin{array}{l}\cdots \cdots \\
\ldots \ldots . . \\
\ldots \ldots\end{array}$ \\
\hline & \multicolumn{9}{|c|}{ (a) } \\
\hline \multirow{3}{*}{$\begin{array}{l}\text { WSLS } \\
\text { WSLS }\end{array}$} & $\cdots \cdots \cdot \mathrm{C}$ & $\mathrm{C}$ & $\mathrm{C}$ & D & $\overline{\mathbf{D}}$ & C & C & C & ...... \\
\hline & $\cdots \cdots \mathrm{C}$ & C & C & C & D & C & C & C & ....... \\
\hline & \multicolumn{9}{|c|}{ (b) } \\
\hline WSLS & $\cdots \cdots C$ & $\mathrm{C}$ & $\mathrm{C}$ & C & $\overline{\mathbf{D}}$ & C & $\bar{C}$ & $\mathbf{C}$ & ..... \\
\hline \multirow[t]{2}{*}{ WSLS } & $\cdots \cdots C$ & C & C & D & D & C & C & C & ....... \\
\hline & \multicolumn{9}{|c|}{ (c) } \\
\hline WSLS & $\cdots \cdots \cdot \mathrm{C}$ & $\mathrm{C}$ & $\mathrm{C}$ & D & $\mathbf{C}$ & C & C & $\mathrm{C}$ & … \\
\hline WSLS & $\cdots \cdots \cdot \mathrm{C}$ & C & C & D & C & C & C & C & ....... \\
\hline
\end{tabular}

(d)

Figure 2 The WSLS strategy.

\begin{tabular}{|c|c|c|c|c|c|c|c|c|c|}
\hline TFT & $\cdots \cdots \cdot C$ & C & $\Gamma$ & C & C & C & C & C & …... \\
\hline TFT & $\cdots \cdots \cdot \mathrm{C}$ & C & C & C & C & C & C & C & ….... \\
\hline
\end{tabular}

(a)

\begin{tabular}{llllllllll|} 
TFT & $\cdots \cdots \cdot$ & C & C & D & C & D & C & D & $\cdots \cdots$ \\
TFT & $\cdots \cdots \cdot$ & C & C & C & D & C & D & C & $\cdots \cdots$ \\
\cline { 2 - 7 }
\end{tabular}

(b)

\begin{tabular}{llllllllll|} 
TFT & $\cdots \cdots \cdot C$ & C & C & C & D & C & D & C & $\cdots \cdots \cdot$ \\
TFT & $\cdots \cdots \cdot C$ & C & C & D & C & D & C & D & $\cdots \cdots \cdot$ \\
\cline { 2 - 6 }
\end{tabular}

(c)

TFT

TFT

\begin{tabular}{|lllllllll|}
\hline$\cdots \cdots \cdot C$ & C & C & D & D & D & D & D & $\cdots \cdots$ \\
$\cdots \cdots \cdot C$ & C & C & D & D & D & D & D & $\cdots \cdots$ \\
\hline
\end{tabular}

(d)

Figure 3 The TFT strategy.

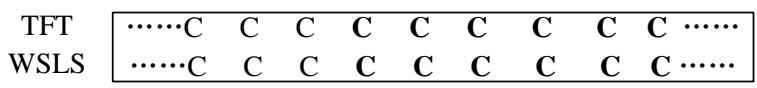

(a)

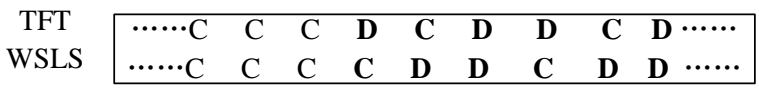

(b)

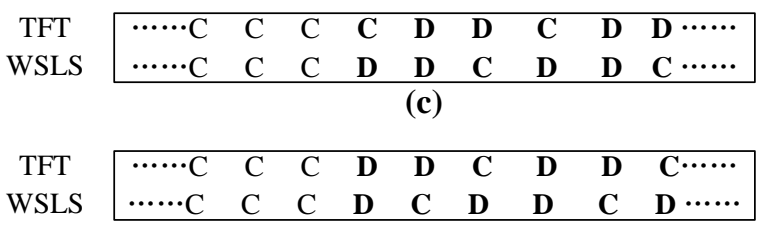

(d)

Figure 4 One party for TFT strategy and one for WSLS.

The basic idea of this paper is as follows. Parties in clouds are considered as rational who have action set and utility function. They are connected in clouds especially a social cloud. In this paper, we take Zachary network as an example and assume that parties belong to this network. As rational parties, they decide whether to share their files in clouds according to their utility. This is the key point which is different from previous works. Sharing files in clouds is considered as cooperating with others, otherwise as defecting from others. This is similar to the process of RSS, where parties decide whether to send his shares to his opponent. The protocol of file sharing consists of multiple rounds just like RSS for parties to interact. The main task in the protocol is how to boost cooperation among parties in clouds. The contributions of this paper are as follows.

- The incentives for parties to share files in clouds are considered using game theory. The seminal idea of this paper is to borrow the notion of rational parties in file sharing on the basis of cloud computing. To the best of our knowledge, utilities are first introduced in file sharing when parties belong to a social cloud.

- Following the basic idea of RSS, we propose the notion of rational file sharing in clouds and its main task is still how to boost cooperation among distrustful parties just like those in RSS. In this paper we use WSLS strategy to replace the commonly used TFT strategy in iterated PD game. Here we take different step in the same direction to encourage cooperation.

- To better illustrate the theory results, we simulate the results at the last section by assuming that parties come from Zachary network. The simulation results show that rational parties are inclined to take cooperation within fewer rounds when they take WSLS strategy than the scenario when they take TFT strategy.

Mutual cooperation brings higher utility for rational parties. That is, the fewer rounds parties need, the higher utility they will get. Therefore WSLS is optimal for rational file sharing in clouds.

\subsection{Organization}

The rest of paper is organized as follows. Section 2 presents some preliminaries such as utility, Nash equilibrium and cloud computing. Section 3 presents a model of file sharing in clouds by using WSLS strategy. Utilities under various conditions are considered in this section according to different strategies they adopt. Section 4 simulates the cooperation level in file sharing, compared with that of using TFT strategy. Zachary network is introduced to better illustrate the relationship of parties in cloud. Suppose parties come from Zachary network and they interact with each other as in a social network using TFT or WSLS strategies. Results show that the cooperation level in the network where parties use WSLS strategy is distinct higher than the network where parties use other strategies including TFT. Section 5 concludes this paper and presents some future works.

\section{Preliminaries}

\subsection{Utility and Nash equilibrium}

Utility definition is the key point in rational scenarios since parties adopt actions according to it. In this paper we follow the utility definition derived from PD game as most RSS settings. For simplicity, we use simultaneous 
Table 1 The utility for rational parties

\begin{tabular}{|c|c|c|}
\hline $\mathrm{P}_{P_{i}} P_{j}$ & Cooperate (C) & Defect (D) \\
\hline Cooperate $(\mathrm{C})$ & $(U, U)$ & $\left(U^{--}, U^{+}\right)$ \\
\hline Defect (D) & $\left(U^{+}, U^{--}\right)$ & $\left(U^{-}, U^{-}\right)$ \\
\hline
\end{tabular}

channels instead of non-simultaneous ones such that utility can be presented in matrix form. Let $\Gamma(P, A, U)$ denote a PD game, where $P=\left\{P_{i}\right\}_{i \in N}$ and $N=\{1,2, \ldots, n\}$ (a finite set of players). Furthermore, let $A=\times_{i \in N} A_{i}$, where $A_{i}$ is a finite action set of player $P_{i}$. In this paper, $A_{i}=$ $\{$ cooperate $(C)$, defect $(D)\}$, where the action cooperate (denoted as $C$ ) means that parties are willing to sharing with others, while the action defect (denoted as $D$ ) means the opposite direction. Let $U=\left\{u_{i}\right\}_{i \in N}$ be the utility set of players. In the setting of rational file sharing, the utility definition should be adjusted to this setting. Let $\mu_{i}(o)$ be the utility of party $P_{i}$ with the outcome $o$, and $\delta_{i}(o)$ be an indicator denoting $P_{i}$ sharing his files with others, and num $(o)=\sum_{i} \delta_{i}(o)$ denote the aggregated number of parties who are willing to share files with $P_{i}$. According to Halpern and Teague (2004) we make the utility function assumptions as:

- $\delta_{i}(o)>\delta_{i}\left(o^{\prime}\right)$, then $\mu_{i}(o)>\mu_{i}\left(o^{\prime}\right)$, i.e., players prefer to share files with others.

- if $\delta_{i}(o)=\delta_{i}\left(o^{\prime}\right)$, then $\operatorname{num}(o)<\operatorname{num}\left(o^{\prime}\right)$, and $\mu_{i}(o)>\mu_{i}\left(o^{\prime}\right)$, i.e., $P_{i}$ prefers strategies in which the fewest number of parties are willing to share files with others.

For two rational parties, there are altogether four outcomes according to the action set.

- $u_{i}=U^{+}$, if $P_{i}$ shares the file while $P_{j}$ does not, $\forall j \neq$ $i$

- $u_{i}=U$, if both $P_{i}$ and $P_{j}$ share the file where $j \neq i$;

- $u_{i}=U^{-}$, if neither $P_{i}$ nor $P_{j}$ shares the file where $j \neq$ $i$

- $u_{i}=U^{--}$, if $P_{j}$ shares the file while $P_{i}$ does not, $\forall j \neq$ $i$;

where $U^{+}>U>U^{-}>U^{--}>0$ holds, otherwise players have no incentive to participate in the protocol for they are rational players acting according to their own benefits (this is much like the scenario in prisoner's dilemma game Axelrod (1990)). Tab. 1 is the utility matrix for two rational parties.

Just like rational secret sharing schemes, the most important notion in rational file sharing is also Nash equilibrium Osborne and Rubinstein (2004) which enables parties not to deviate from the protocol so that the construction can be completed. Roughly speaking, a protocol induces a Nash equilibrium if no party gains any advantages by deviating from the protocol, as long as all other parties follow the protocol. Halpern and Teague suggest to design protocols that induce a Nash equilibrium surviving
IDOWDS which is used in subsequent work of Abraham et al. (2006); Gordon and Katz (2006); Lysyanskaya and Triandopoulos (2006). Researchers propose various schemes to overcome the drawbacks of IDOWDS such as everlasting equilibrium and strict Nash equilibrium Katz (2008); Kol and Naor (2008a,b). We follow the definitions in game theory scenario where $\vec{\sigma} \stackrel{\text { def }}{=}\left(\sigma_{1}, \ldots, \sigma_{i-1}, \sigma_{i}, \sigma_{i+1}, \ldots, \sigma_{n}\right)$, $\vec{\sigma}_{-i} \stackrel{\text { def }}{=}\left(\sigma_{1}, \ldots, \sigma_{i-1}, \sigma_{i+1}, \ldots, \sigma_{n}\right) \quad$ and $\quad\left(\sigma_{i}^{\prime}, \vec{\sigma}_{-i}\right) \stackrel{\text { def }}{=}$ $\left(\sigma_{1}, \ldots, \sigma_{i-1}, \sigma_{i}^{\prime}, \sigma_{i+1}, \ldots, \sigma_{n}\right) .\left(\sigma_{i}^{\prime}, \vec{\sigma}_{-i}\right)$ denotes the strategy vector $\vec{\sigma}$ with $P_{i}$ adopting $\sigma_{i}^{\prime}$ while others obey the specified strategies assigned by $\vec{\sigma}$.

We say $\sigma_{i} \in A_{i}$ weakly dominates $\sigma_{i}^{\prime} \in A_{i}$ relative to $\vec{\sigma}_{-i}$ if

$$
u_{i}(\vec{\sigma}) \geq u_{i}\left(\sigma_{i}^{\prime}, \vec{\sigma}_{-i}\right)
$$

Then $\vec{\sigma}$ is a Nash equilibrium if, for all $i$, the action $\sigma_{i}$ weakly dominates all actions in $A_{i}$ relative to $\vec{\sigma}_{-i}$. We give formal definition of Nash equilibrium.

Definition 2.1: A strategy $\vec{\sigma}$ induces a Nash equilibrium if for any player $P_{i}$ and any strategy $\sigma_{i}^{\prime}$, it holds that

$$
u_{i}\left(\sigma_{i}^{\prime}, \sigma_{-i}\right) \leq u_{i}(\vec{\sigma})
$$

\subsection{Cloud computing}

Cloud computing is called the fifth utility together with electricity, water, telephony and gas Buyya et al. (2009), which has been changing the way computer resources have been allocated and assigned. Consumers accessed the utilities and paid for the service without concerning the way the services generated. Cloud computing presents a paradigm in the way of computing resources acquisition. Before the advent of cloud computing, users either purchase outright or leased from data center in order to get computing resources at fixed rate no matter how may resources you utilized. Now with the convenient brought by cloud computing, users can access the computing services in a similar way and pay only for the services they accessed. Cloud computing consists of cloud service providers and customers. The former ones host their services on the Internet and provide them to the prospective customer. The latter ones may access these services when needed and only pay for the accessed service. Through virtualized systems, service providers can sold the same resource to different customers such that they can achieve maximum utilization fro the resources. On the other hand, the customers using virtualized system feel like that the resources are belonged to them although in fact the resources are shared among various customers de Assuncao et al. (2009). Fig. 5 presents the architecture of cloud computing. It can be seen that cloud computing consists of five layers: software as a service (SaaS) layer, platform as a service (PaaS) layer, infrastructure as a service (IaaS) layer Vecchiola et al. (2009), virtualized hardware layer and physical hardware layer.

SaaS models the software deployment over the Internet. IaaS provides raw computer infrastructure in terms of virtual computers in clouds computing. PssS provides facilities and applications programming interfaces (API) to support 


\begin{tabular}{|c|}
\hline Software as a Service (SaaS) Layer \\
\hline Platform as a Service (PaaS) Layer \\
\hline Infrastructure as a Service (IaaS) Layer \\
\hline Virtualized Hardware Layer \\
\hline Physical Hardware Layer \\
\hline
\end{tabular}

Figure 5 The architecture of cloud computing.

the whole life cycle of web applications and services. The virtualized hardware layer and physical hardware layer are made up by server class computers in data centers, clusters, grids, storage networks etc.

\section{File sharing in clouds with WSLS}

As Maleka et al. (2008), $\Gamma(n, m)$ denotes the infinitely repeated PD game where $n$ is the number of parties and $m$ is the parties' set who participate in the game to share files. In this paper, we consider that parties belong to the cloud computing. When one party shares files with others, he is considered as a resource providers and the receiver is considered as a consumer who will receive the resource. Therefore, the protocol of file sharing can be regarded as cloud computing. In $\Gamma(n, m)$, each party may choose the other party in clouds computing to share his files. However, in previous RSS schemes, no party is willing to share his files with his opponent. Although we have introduced TFT strategy into file sharing in our previous works, TFT still has some shortcomings. Thus, in this paper, we introduce WSLS strategy to overcome these shortcomings. As shown in section 1 , WSLS is robust for noise and evolutionarily stable. Section 1 only shows how two parties interact when they adopt TFT and WSLS strategies respectively. Here we will show how they interact in clouds computing.

We first analyze the simplest case, where only two parties in file sharing and assume that at the first round of the infinite file sharing parties both cooperate with each other. Fig. 6 presents the fame for parties in cloud. In Fig. 6, the dotted line denotes that it seems two parties decide whether to share files with the other party. In fact, the real interaction between them is through cloud. Then we will discuss a complex case, where a number of parties participate in file sharing and they may adopt cooperation or defect according to their groups. If no parties deviate from cooperation, then both parties will cooperate till the end of the file sharing. Note that we only discuss infinite file sharing in this paper and leave the file sharing case for future works.

As the infinite PD game, there is a discount factor $\delta \in(0,1)$ when computing the utility. For example, one utility today may decrease to $\delta$ tomorrow, $\delta^{2}$ the day after tomorrow and so on. When no parties deviate, both parties

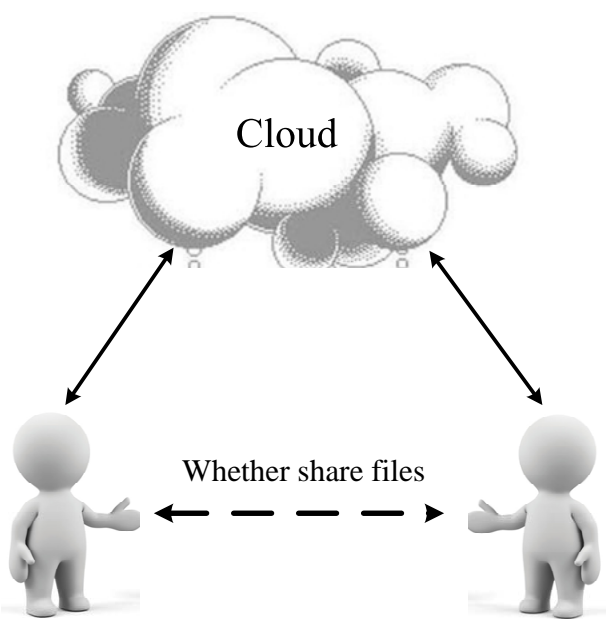

Figure 6 The fame for parties in cloud.

will cooperate in the file sharing. The total utility $U_{\text {nodeviation }}$ for both parties is denoted in Equation (1):

$$
U+\delta U+\delta^{2} U+\delta^{3} U+\ldots=\frac{U}{1-\delta} .
$$

In Fig. 7, both parties adopt TFT strategy and the second party deviates at the $(t+1)^{t h}$ round hoping to achieve a better utility. When the second party deviates at the $(t+1)^{t h}$ round, the first party will defect at the $(t+2)^{t h}$ round and the second party will cooperate since the first party cooperates at the $(t+1)^{t h}$ round. At the $(t+3)^{t h}$ round, the first party cooperates and the second party defects according to the rules of TFT strategy. Therefore, both parties alternately cooperate and defect at the following rounds. To analyze whether parties can achieve better utility through deviating, we only discuss the utility of the deviating party. The second party will obtain $U$ during the first $t$ rounds, $U^{+}$at the $(t+1)^{t h}$ round, $U^{--}$ at the $(t+2)^{t h}$ round, $U^{+}$at the $(t+3)^{t h}$ round and so on. The total utility $U_{T F T}$ for this case considering the discount factor is denoted in Equation (2):

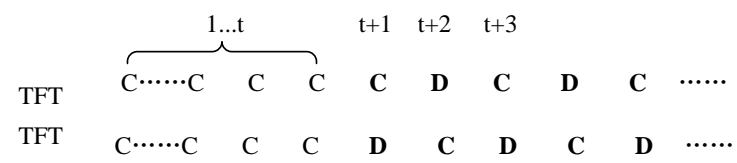

Figure 7 One party deviates when both of them adopt TFT.

$$
\begin{aligned}
& U+\ldots+\delta^{t} U+\delta^{t+1} U^{+}+\delta^{t+2} U^{--}+\delta^{t+3} U^{+}+\ldots \\
& =U+\ldots+\delta^{t} U+\left(\delta^{t+1} U^{+}+\delta^{t+3} U^{+} \ldots\right) \\
& +\left(\delta^{t+2} U^{--}+\delta^{t+4} U^{--}+\ldots\right) \\
& =\frac{U\left(1-\delta^{t}\right)}{1-\delta}+\delta^{t+1} U^{+} \frac{1}{1-\delta^{2}}+\delta^{t+2} U^{--} \frac{1}{1-\delta^{2}}
\end{aligned}
$$

In Fig. 8, both parties adopt WSLS strategy and the second party deviates at the $(t+1)^{t h}$ round hoping to achieving a better utility. When the second party deviates at the $(t+$ $1)^{t h}$ round, the first party will defect at the $(t+2)^{t h}$ round, which is the opposite action at the $(t+1)^{t h}$ round, since the 
outcome at the $(t+1)^{t h}$ round is $(C, D)$ for him. On the other hand, the second party will continue defect, which is the same action to the $(t+1)^{t h}$ round, since the the outcome at the $(t+1)^{t h}$ round is $(D, C)$ for him. Since the outcome at the $(t+2)^{t h}$ round is $(D, D)$, both parties will cooperate at the $(t+3)^{t h}$ round according to the rules of WSLS strategy. Therefore in the following rounds, both parties will turn back to mutual cooperation. The second party will obtain $U$ during the first $t$ rounds, $U^{+}$at the $(t+1)^{t h}$ round, $U^{-}$at the $(t+$ $2)^{t h}$ round, $U$ at the $(t+3)^{t h}$ round and $U$ in the following rounds. The total utility $U_{W S L S}$ for this case considering the discount factor is denoted in Equation (3):

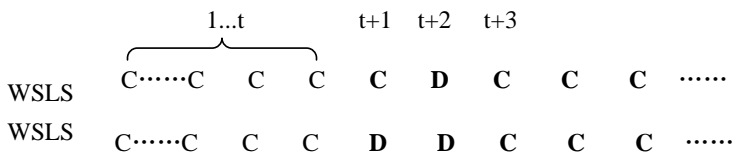

Figure 8 One party deviates when both of them adopt WSLS.

$$
\begin{aligned}
U & +\ldots+\delta^{t} U+\delta^{t+1} U^{+}+\delta^{t+2} U^{-}+\delta^{t+3} U+\ldots \\
= & U+\ldots+\delta^{t} U+\left(\delta^{t+1} U+\delta^{t+2} U\right)+\delta^{t+3} U+\ldots \\
& +\delta^{t+1} U^{+}+\delta^{t+2} U^{-}-\left(\delta^{t+1} U+\delta^{t+2} U\right) \\
= & \frac{U}{1-\delta}+\delta^{t+1}\left(U^{+}-U\right)+\delta^{t+2}\left(U^{-}-U\right) .
\end{aligned}
$$

In Fig. 9, the first party adopts TFT strategy and the second party adopts WSLS strategy. The second party deviates at the $(t+1)^{t h}$ round hoping to achieve a better utility. When the second party deviates at the $(t+1)^{t h}$ round, the first party will defect at the $(t+2)^{t h}$ round since the first party will adopt the action which the second party adopts at the $(t+1)^{t h}$ round. The second party will continue to defect at the $(t+2)^{t h}$ round, which is the same action to the $(t+1)^{t h}$ round, since the the outcome at the $(t+1)^{t h}$ round is $(D, C)$ for him. The first party will continue to defect at the $(t+3)^{t h}$ round since the second party defects at the $t+2^{t h}$ round. While the second party will change to cooperate since the outcome of the $(t+2)^{t h}$ round is $(D, D)$. Consequently, both party will enter into a circulation consisting three outcomes $(C, D),(D, D,(D, C)$, as shown in the shadow parts in Fig. 9. The second party will obtain $U$ during the first $t$ rounds, $U^{+}$at the $(t+1)^{t h}$ round, $U^{-}$at the $(t+2)^{t h}$ round, $U^{--}$at the $(t+3)^{t h}$ round and so on. The total utility $U_{T F T W S L S}$ for this case considering the discount factor is denoted in Equation (4):

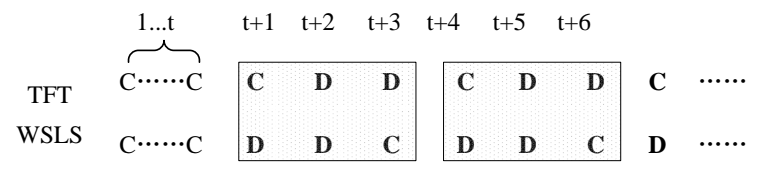

Figure 9 One party deviates when one adopts WSLS and one adopts TFT.

$$
\begin{aligned}
& U+\ldots+\delta^{t} U+\delta^{t+1} U^{+}+\delta^{t+2} U^{-}+\delta^{t+3} U^{--} \\
& +\delta^{t+4} U^{+}+\delta^{t+5} U^{-}+\delta^{t+6} U^{--}+\ldots \\
& =U+\ldots+\delta^{t} U+\left(\delta^{t+1} U^{+}+\delta^{t+4} U^{+}+\ldots \ldots\right) \\
& +\left(\delta^{t+2} U^{-}+\delta^{t+5} U^{-}+\ldots \ldots\right) \\
& +\left(\delta^{t+3} U^{--}+\delta^{t+6} U^{--}+\ldots \ldots\right) \\
& =\frac{U}{1-\delta}+\delta^{t+1} \frac{U^{+}}{1-\delta^{3}}+\delta^{t+2} \frac{U^{-}}{1-\delta^{3}}+\delta^{t+3} \frac{U^{--}}{1-\delta^{3}} .
\end{aligned}
$$

\section{Simulation results}

To analyze whether the second party achieves a better utility by deviating, we draw the utility functions in Equation (1)(2)(3)(4), where $\delta \in(0,1)$. Given $U^{+}=3, U=2, U^{-}=$ $1, U^{--}=0$ and $t=1$, which mean that party deviates at the second round, we have

$$
\begin{aligned}
& U_{\text {nodeviation }}=\frac{2}{1-\delta}, \\
& U_{T F T}=2+\frac{3 \delta^{2}}{1-\delta^{2}}, \\
& U_{W S L S}=\frac{2}{1-\delta}+\delta^{2}-\delta^{3}, \\
& U_{T F T W S L S}=2+\frac{3 \delta^{2}+\delta^{3}}{1-\delta^{3}} .
\end{aligned}
$$

Since $\delta \in(0,1), \quad$ it satisfies that $U_{W S L S}>$ $U_{\text {nodeviation }}>U_{T F T}>U_{T F T W S L S}$. Fig. 10 presents the relationships among the utility functions.

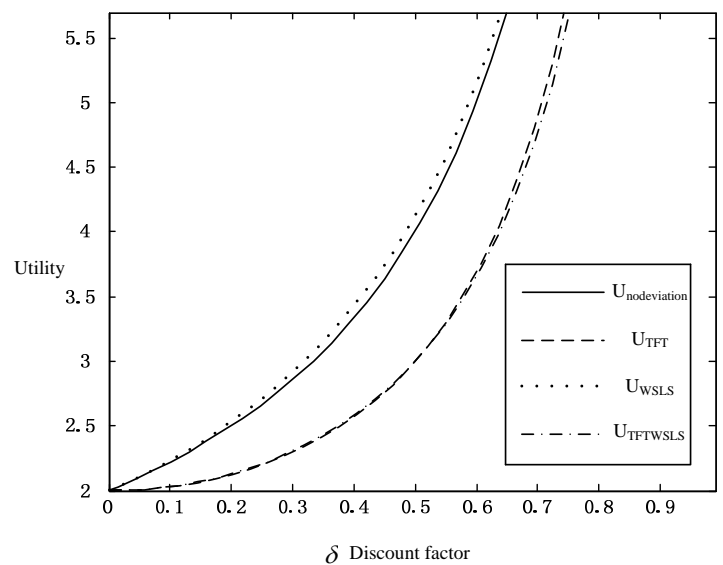

Figure 10 The comparison among utility functions.

$U_{\text {nodeviation }}$ is the base line where no parties deviate. According to Fig. 10, $U_{W S L S}$ is a little bit higher than the base line, While $U_{T F T}$ and $U_{T F T W S L S}$ is lower than the baseline. Therefore, we conclude that WSLS strategy can bring higher utility than other strategies where exist a mistake deviation. That is, if both parties take WSLS strategy, the outcome will finally come back to mutual cooperation even if one party deviates accidentally. Note that if at least one party takes TFT strategy, both parties can not come back to mutual cooperation even if the other party takes WSLS strategy. 
In the above sections, we discuss the settings where only two parties interact. The simulation results show that if both parties take WSLS, they will come back to mutual cooperation even if there are some mistake deviations. In the following sections, we will discuss how parties interact in cloud. Here, game theory is introduced into cloud computing to encourage parties to share their files in cloud. Here, sharing files with others is deemed as cooperating with others and not sharing files with others is deemed as defecting from others. Then file sharing in clouds can be converted as a game mentioned in section 1 . Moreover, the utility definitions for parties in clouds to share file are the same to Tab. 1. Therefore, we abuse the notions of cooperation and defect in file sharing in clouds to denote sharing and not sharing respectively.

It's more complex to discuss multi-party case than twoparty case. There are some key problems to solve.

1. How these parties connect in cloud. Are they connected in a complete network.

2. How to initialize the action at the beginning round.

3. How to make out a mistake deviation or an intentional deviation.

To solve the first problem, Zachary network is introduced to present the connections of parties in cloud. Zachary network is a classical problem in social network, which present the relationship of university Karate clubs Zachary (1977). This is an incomplete network, which means that not all parties connect with others. To solve the second problem, we initialize parties' actions according to which community they belongs to, since Zachary network is often used in the study of community structures. More specifically, Zachary network is first divided into several communities and initial actions chosen from $C$ or $D$ are assigned to each community such that parties in the same community have the same initial actions. To solve the third problem, we do not distinguish a mistake deviation and an intentional deviation since they bring no discrimination for rational parties. Therefore, they are called deviation as an integral.

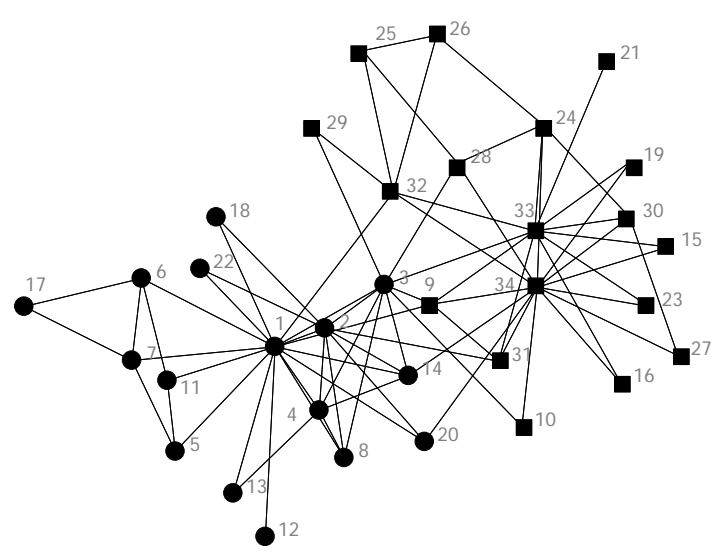

Figure 11 The Zachary network.

The cloud is a dynamical system, so file sharing can be deemed as an infinite process, which includes infinite rounds.
In each round, parties decide whether to share files with others according to his strategy and utilities. The procedure is as follows.

1. To present an incomplete network like Zachary network using an adjacent matrix, where 1 denotes that two parties are connected and 0 denotes that they are not. When two parties connected in the network, they are called neighbors.

2. Divide the network into several communities using the clustering algorithm $k$ Means Cluster, then randomly assign initial actions to each communities. Parties in the same community are assigned to the initial action of the community.

3. In each round, each party interacts with his neighbors using TFT or WSLS strategies.

Fig.12 presents the distribution of each pair of neighbors when both parties adopt TFT strategy. Fig.13 presents the distribution of each pair of neighbors when one party adopts TFT strategy while the other adopts WSLS strategy. Fig.14 presents the distribution of each pair of neighbors when both parties adopt WSLS strategy. In these figures, the point $(x, y, z)$ denote the outcomes of each neighbor, where $z$ denotes the index of each party. Note that there are altogether 34 parties in Zachary network. $(x, y) \quad(x, y \in\{0,1,2\})$ denotes the outcome, where 0 denotes the outcome when two parties are not neighbors, 1 denotes the action of cooperation and 2 denotes the action of defect. round $=i$ means it is the result at the $i^{(t h)}$ round. The sub-figures (a) in Fig. 12, Fig. 13 and Fig.14 present the initial outcomes for each neighbor. Fig. 12 (b)(c)(d) present the outcomes when round is 3,50,100 respectively. From these figures, it can be concluded that TFT can not lead parties to come back to mutual cooperation. Moreover, the outcomes maintain unchanged from the third round. Fig. 13 (b)(c)(d) present the outcomes when round is $3,50,100$ respectively. From these figures, it can be concluded that when one party adopts TFT strategy, parties can not reach mutual cooperation. Distributions of the outcomes are various in Fig. 13 (b)(c)(d). Fig. 14 (b)(c)(d) present the outcomes when round is 3,50,100 respectively. From these figures, it can be concluded that WSLS can lead parties to come back to mutual cooperation. The outcomes maintain to be $(C, C)$ from the third round.

The simulation results show that WSLS strategy makes parties adopt cooperation in fewer rounds. That is, parties will get higher utility when they both take WSLS rather than other strategies like TFT. The reason lies in that mutual cooperation brings higher utility in the long run. Note that although parties will get a highest utility when he defects while his opponent cooperates, the winning part will be systematically offset when considering discount factor. Therefore, it is better for rational parties in file sharing to take WSLS for the sake of long-term utility. In consequence, parties are recommended to take WSLS strategy in rational file sharing in cloud. 


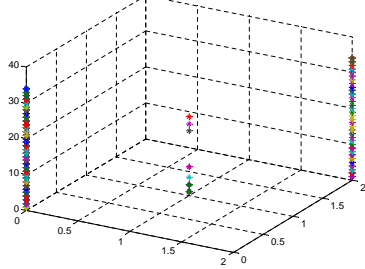

(a) Round $=1$

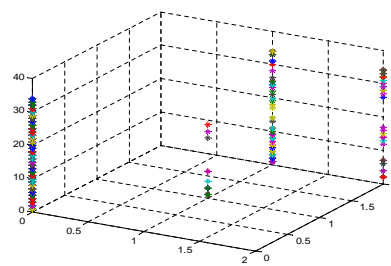

(c) Round $=50$

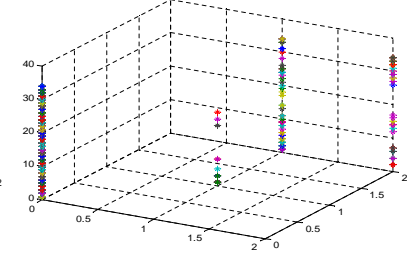

(b) Round $=3$

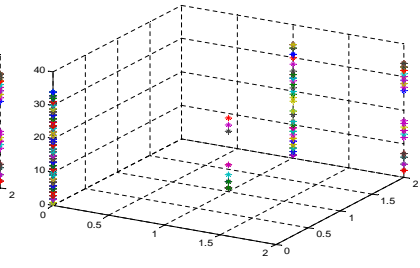

(d) Round $=100$
Figure 12 The simulation results when both parties adopt TFT strategy.

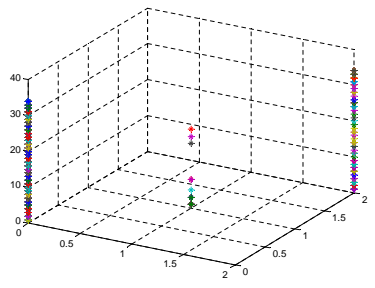

(a) Round $=1$

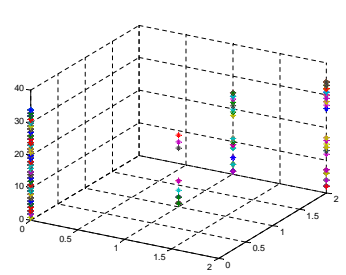

(c) Round=50

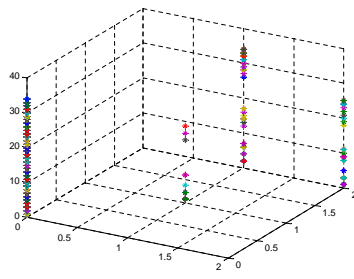

(b) Round=3

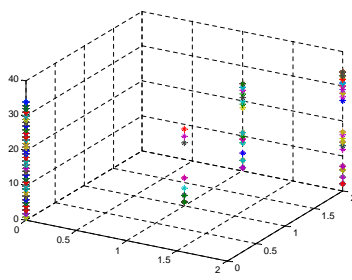

(d) Round $=100$
Figure 13 The simulation results when one party adopts TFT strategy and the other adopts WSLS.

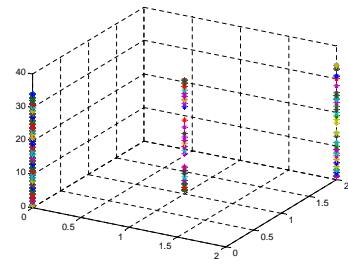

(a) Round $=1$

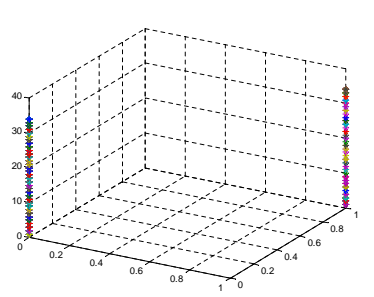

(c) Round $=50$

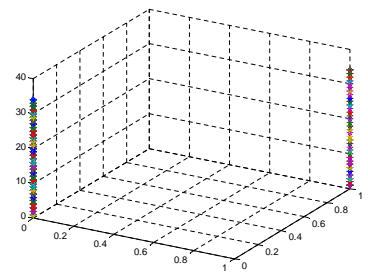

(b) Round=3

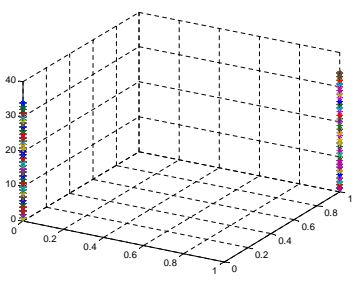

(d) Round=100
Figure 14 The simulation results when both parties adopt WSLS strategy.

\section{Conclusions}

The concept of cloud computing has been evolving for more than 40 years. The underlying premise of cloud computing was a global interconnection of computer programs and data. File sharing in clouds is crucial to much collaborative work, which aims to encourage parties in clouds to cooperate. In this paper, game theory is introduced to give parties incentives such that they are willing to share their files in cloud. Parties in file sharing decide to share files with others on the condition that sharing files can maximize their utilities. Note that the utility definition derives from PD game just like that of RSS, where two assumptions are satisfied. More especially, file sharing in clouds can be considered as infinitely repeated PD game and use the strategies therein to encourage parties to cooperate such that they can maximize their utilities. As often used in infinitely repeated PD game, WSLS strategy is considered here to boost cooperation among parties in cloud. Comparing to TFT strategy, WSLS can better deal with unintentional defect in file sharing. That is, parties using WSLS strategy can come back to mutual cooperation when there is an unintentional defect, while TFT strategy can not. We simulate the process of file sharing to illustrate the effect of WSLS on the utility. Simulation results show that parties can achieve higher utilities when all of them take WSLS strategy. Here we only consider the settings where parties are rational. In the future works, various kinds of parties like malicious parties should be considered to discuss stronger security guarantee. Furthermore, the settings allowing collusion among a set of rational parties should also be discussed in the future works.

\section{Acknowledgements}

This work was supported by the Natural Science Foundation of China under Grant No. 61173139, Natural Science Foundation of Shandong Province under Grant No.ZR2011FZ005, Doctoral Fund of Ministry of Education of China under Grant No. 20110131110027, Independent Innovation Foundation of Shandong University under Grant No. 2012TS073 and Open Research Fund of Beijing Key Laboratory of Trusted Computing.

\section{References}

Abraham, I., D. Dolev, R. Gonen, and J. Halpern (2006). Distributed computing meets game theory: robust mechanisms for rational secret sharing and multiparty com- putation. In 25th ACM Symposium Annual on Principles of Distributed Com- puting, New York, NY, USA, pp. 53-62. ACM.

Altman, E., T. Boulogne, R. El-Azouzi, T. Jimenez, and L. Wynter (2006). A survey on networking games in telecommunications. Comput. Oper. Res. 33(2), 286311.

Axelrod, R. (1990). The Evolution of Cooperation. Penguin Press. 
Buyya, R., C. S. Yeo, S. Venugopal, J. Broberg, and I. Brandic (2009). Cloud computing and emerging it platforms: Vision, hype, and reality for delivering computing as the 5th utility. Journal of Future Generation Computer Systems 25(6), 599-616.

Canedo, E., R. de Oliveira Albuquerque, and R. T. de Sousa Junior (2011). Trust model for file sharing in cloud computing. In The Second International Conference on Cloud Computing, GRIDs, and Virtualization, ACM, pp. 66-73.

Carroll, T. and D. Grosu (2011). A game theoretic investigation of deception in network security. Security and Communication Networks 4(10), 1162-1172.

Christin, N., A. S. Weigend, and J. Chuang (2005). Content availability, pollution and poisoning in file sharing peertopeer networks. In Proc. EC 2005, ACM, pp. 68-77.

de Assuncao, M., A. di Costanzo, and R. Buyya (2009). Evaluating the cost-benefit of using cloud computing to extend the capacity of clusters. In 18th ACM international symposium on High performance distributed computing (HPDC '09), Munich, Germany, pp. 141-150.

Ficco, M. (2013). Security event correlation approach for cloud computing. International Journal of High Performance Computing and Networking 7(3), 173-185.

Ge, Y., Y. Zhang, Q. Qiu, and Y. H. Lu (2012). A game theoretic resource allocation for overall energy minimization in mobile cloud computing system. In Proceedings of the 2012 ACM/IEEE international symposium on Low power electronics and design, New York, NY, USA, pp. 279-284. ACM.

Gordon, S. and J. Katz (2006). Rational secret sharing, revisited. In SCN 2006, Heidelberg, pp. 229-241. Springer.

Halpern, J. and V. Teague (2004). Rational secret sharing and multiparty computation: extended abstract. In STOC '04: Proceedings of the thirty-sixth annual ACM symposium on Theory of computing, New York, NY, USA, pp. 623-632. ACM.

Hu, H., L. Z, and H. Hu (2012). An anti-cheating bidding approach for resource allocation in cloud computing environments. Journal of Computational Information Systems 8(4), 1641-1654.

Johnson, M., D. McGuire, and N. D. Willey (2009). Why file sharing networks are dangerous? J. Commun. ACM 52(2), $134-138$

Katz, J. (2008). Bridging game theory and cryptography: Recent results and future directions. In 5th Theory of Cryptography Conference TCC 2008, pp. 251-272. Springer.

Kol, G. and M. Naor (2008a). Cryptography and game theory: Designing protocols for exchanging infor- mation. In Canetti, R. (ed.) TCC 2008, Heidelberg, pp. 320-339. Springer.
Kol, G. and M. Naor (2008b). Games for exchanging information. In STOC 2008, New York, NY, USA, pp. 423432. ACM.

Li, W., J. Liang, and Z. Xu (2004). Vegafs: file sharing crossing multiple domains. International Journal of High Performance Computing and Networking 2(3), 178-185.

Lo, C. C., C. C. Huang, and J. Ku (2010). A cooperative intrusion detection system framework for cloud computing networks. In Parallel Processing Workshops (ICPPW), pp. 280-284. IEEE.

Lysyanskaya, A. and N. Triandopoulos (2006). Rationality and adversarial behavior in multi-party computation. In Dwork, C. (ed.) CRYPTO 2006, Heidelberg, pp. 180-197. Springer.

Maleka, S., A. Shareef, and C. P. Rangan (2008). Rational secret sharing with repeated games. In ISPEC'08 Proceedings of the 4th international conference on Information security practice and experience, Heidelberg, pp. 334-346. Springer.

Micali, S. and A. Shelat (2009). Purely rational secret sharing (extended abstract). In 6th Theory of Cryptography Conference, pp. 54-71.

Muller, M. and J. Feinberg (2010). Information curators in an enterprise file-sharing service. In Proc. ECSCW 2009, pp. 403-410. ACM Press.

Muller, M., D. R. Millen, and J. Feinberg (2010). Patterns of usage in an enterprise file-sharing service: publicizing, discovering, and telling the news. In Proc. CHI 2010, pp. 763-766. ACM Press.

Niyato, D., A.V.Vasilakos, and Z. Kun (2011). Resource and revenue sharing with coalition formation of cloud providers: Game theoretic approach. In Cluster, Cloud and Grid Computing (CCGrid), pp. 215-224. IEEE.

Nowak, M. and K. Sigmund (1993). A strategy of win-stay, lose-shift that outperforms tit for tat in prisoner's dilemma. Nature 364, 56-58.

Osborne, M. and A. Rubinstein (2004). A Course in Game Theory. Cambridge: MIT Press.

Rader, E. (2009). Yours, mine and (not) ours: social influences on group information repositories. In Proc. CHI 2009, pp. 2095-2098. ACM Press.

Rader, E. (2010). The effect of audience design on labeling, organizing, and finding shared files. In Proc. CHI 2010, pp. 777-786. ACM Press.

Roughgarden, T. (2010). Algorithmic game theory. Commun. ACM 53(7), 7886.

Schwartz, A. Z. (2007). Ud dropbox 2.0: collaboration magic. In Proc. SIGUCCS 2007, pp. 305-309. ACM Press. 
Shami, N., M. Muller, and D. Millen (2011). Browse and discover: social file sharing in the enterprise. In Proceedings of the ACM 2011 conference on Computer supported cooperative work, pp. 295-304. ACM Press.

Tang, J. C., C. Drews, M. Smith, F. Wu, A. Sue, and T. Lau (2007). Exploring patterns of social commonality among file directories at work. In In Proc. CHI 2007, pp. 951-960. ACM Press.

Vecchiola, C., S. Pandey, and R. Buyya (2009). Highperformance cloud computing: A view of scientific applications. In 10th International Symposium on Pervasive Systems, Algorithms and Networks, Kaohsiung, Taiwan, pp. 4-16.

Voida, S., W. K. Edwards, M. W. Newman, R. E. Grinter, and N. Ducheneaut (2006). Share and share alike: Exploring the user interface affordances of file sharing. In Proc. CHI 2006, pp. 221-230. ACM Press.

Wang, J., P. Varman, and C. Xie (2010). Middleware enabled data sharing on cloud storage services. In Proceedings of the 5th International Workshop on Middleware for Service Oriented Computing, pp. 33-38. ACM Press.

Wang, Y., Z. Liu, H. Wang, and Q. Xu (2013). Social rational secure multi-party computation. Concurrency and Computation: Practice and Experience 26(5), 1076-1083.

Wang, Y., Z. Liu, and Q. Xu (2014). New rational parties relying on reputation. Security and Communication Networks 7, 11281137.

Wang, Y., H. Wang, and Q. Xu (2012a). Rational secret sharing with semi-rational players. International Journal of Grid and Utility Computing 3(1), 59-67.

Wang, Y., H. Wang, and Q. Xu (2012b). Repeated rational secret sharing, revisited. International Journal of Grid and Utility Computing 3(1), 12-24.

Whalen, T., E. G. Toms, and J. Blustein (2008). Information displays for managing shared files. In Proc. CHIMIT 2008, pp. 1-10. ACM Press.

Xiao, P. and N. Han (2014). A novel power-conscious scheduling algorithm for data-intensive precedenceconstrained applications in cloud environments. International Journal of High Performance Computing and Networking 7(4), 299-306.

Xu, L., X. Wu, and X. Zhang (2012). Cl-pre: a certificateless proxy re-encryption scheme for secure data sharing with public cloud. In Proceedings of the 7th ACM Symposium on Information, Computer and Communications Security, pp. 87-88. ACM Press.

Yang, D., X. Fang, and G. Xue (2012). Game theory in cooperative communications. Wireless Communications 19(2), 44-49.
Zachary, W. W. (1977). An information flow model for conflict and fission in small groups. Journal of Anthropological Research 33, 452473.

Zhou, M., R. Zhang, D. Zeng, and W. Qian (2010). Services in the cloud computing era: a survey. In 4th International Software Engineering Institute. Universal Communication. Symposium (IUCS), Shanghai, China, pp. 40-46. 